\title{
Corrigendum: Retinal lipid and glucose metabolism dictates angiogenesis through the lipid sensor Ffar1
}

Jean-Sébastien Joyal, Ye Sun, Marin L Gantner, Zhuo Shao, Lucy P Evans, Nicholas Saba, Thomas Fredrick, Samuel Burnim, Jin Sung Kim, Gauri Patel, Aimee M Juan, Christian G Hurst, Colman J Hatton, Zhenghao Cui, Kerry A Pierce, Patrick Bherer, Edith Aguilar, Michael B Powner, Kristis Vevis, Michel Boisvert, Zhongjie Fu, Emile Levy, Marcus Fruttiger, Alan Packard, Flavio A Rezende, Bruno Maranda, Przemyslaw Sapieha, Jing Chen, Martin Friedlander, Clary B Clish \& Lois E H Smith Nat. Med.; doi:10.1038/nm.4059; corrected 24 March 2016

In the version of this article initially published online, there were two errors. There was a typographical error in the text, which should have stated that the 'dark current' is an electrochemical gradient required for photon-induced polarization (rather than depolarization, as incorrectly stated). In addition, some funding sources were inadvertently omitted from the Acknowledgments. The errors have been corrected for the print, PDF and HTML versions of this article.

\section{Corrigendum: ROR- $\gamma$ drives androgen receptor expression and represents a therapeutic target in castration-resistant prostate cancer}

Junjian Wang, June X Zou, Xiaoqian Xue, Demin Cai, Yan Zhang, Zhijian Duan, Qiuping Xiang, Joy C Yang, Maggie C Louie, Alexander D Borowsky, Allen C Gao, Christopher P Evans, Kit S Lam, Jianzhen Xu, Hsing-Jien Kung, Ronald M Evans, Yong Xu \& Hong-Wu Chen

Nat. Med.; doi:10.1038/nm.4070; corrected online 22 April 2016

In Figure 2a of the version of this article initially published online, one phenyl ring was inadvertently deleted from the chemical structure of compound SR2211. One affiliation of H.-W.C. (Veterans Affairs Northern California Health Care System-Mather, Mather, California, USA) was also inadvertently omitted. These errors have been corrected for the print, PDF and HTML versions of this article.

\section{Corrigendum: Protection against malaria at 1 year and immune correlates following PfSPZ vaccination}

Andrew S Ishizuka, Kirsten E Lyke, Adam DeZure, Andrea A Berry, Thomas L Richie, Floreliz H Mendoza, Mary E Enama, Ingelise J Gordon, Lee-Jah Chang, Uzma N Sarwar, Kathryn L Zephir, LaSonji A Holman, Eric R James, Peter F Billingsley, Anusha Gunasekera, Sumana Chakravarty, Anita Manoj, MingLin Li, Adam J Ruben, Tao Li, Abraham G Eappen, Richard E Stafford, Natasha K C, Tooba Murshedkar, Hope DeCederfelt, Sarah H Plummer, Cynthia S Hendel, Laura Novik, Pamela J M Costner, Jamie G Saunders, Matthew B Laurens, Christopher V Plowe, Barbara Flynn, William R Whalen, J P Todd, Jay Noor, Srinivas Rao, Kailan Sierra-Davidson, Geoffrey M Lynn, Judith E Epstein, Margaret A Kemp, Gary A Fahle, Sebastian A Mikolajczak, Matthew Fishbaugher, Brandon K Sack, Stefan H I Kappe, Silas A Davidson, Lindsey S Garver, Niklas K Björkström, Martha C Nason, Barney S Graham, Mario Roederer, B Kim Lee Sim, Stephen L Hoffman, Julie E Ledgerwood \& Robert A Seder, for the VRC 312 and VRC 314 Study Teams

Nat. Med.; doi:10.1038/nm.4110; corrected online 18 May 2016

In the version of this article initially published online, the authors omitted a funding source, The Bill and Melinda Gates Foundation (Investment ID: 24922). The error has been corrected for the print, PDF and HTML versions of this article.

\section{Erratum: Modulation of splicing catalysis for therapeutic targeting of leukemia with mutations in genes encoding spliceosomal proteins}

Stanley Chun-Wei Lee, Heidi Dvinge, Eunhee Kim, Hana Cho, Jean-Baptiste Micol, Young Rock Chung, Benjamin H Durham, Akihide Yoshimi, Young Joon Kim, Michael Thomas, Camille Lobry, Chun-Wei Chen, Alessandro Pastore, Justin Taylor, Xujun Wang, Andrei Krivtsov, Scott A Armstrong, James Palacino, Silvia Buonamici, Peter G Smith, Robert K Bradley \& Omar Abdel-Wahab Nat. Med.; doi:10.1038/nm.4097; corrected 11 May 2016

In the version of this article initially published online, the graphs in Figure $3 \mathrm{~b}-\mathrm{d}$ were laid out incorrectly and were not consistent with the figure legend and text. The error has been corrected for the print, PDF and HTML versions of this article. 